\title{
Análise Numérica do Comportamento de Pavimentos Constituídos de Lajes Lisas de Concreto Protendido
}

\author{
CUBAS, Vanessa La Torre ${ }^{1}$ \\ NARVAEZ, Nathaly Sarasty² \\ ${ }^{10}$ Pontifícia Universidade Católica do Rio de Janeiro, Brasil. mvlatorrec@gmail.com \\ ${ }^{2}$ Centro Universitário de Brasília, Brasil. nathalysarasty@hotmail.com
}

\begin{abstract}
Resumo
Lajes lisas de concreto protendido com cordoaIhas engraxadas não aderentes têm sido empregadas em pavimentos de edificações com frequência nos últimos anos. Essa solução estrutural é ideal quando se tem uma distribuição regular dos pilares. Além disso, sabe-se que lajes de concreto protendido oferecem vantagens técnicas sobre a solução tradicional em concreto armado, principalmente para vencer vãos maiores e onde muitas vezes se exigem seções mais esbeltas. O objetivo desta pesquisa é estabelecer critérios práticos para o projeto de lajes lisas protendidas, maciças ou nervuradas, visando ao atendimento dos critérios relativos ao estado limite de utilização. Com este propósito, um estudo paramétrico foi realizado, no qual foram analisadas as tensões nas regiões de introdução das forças de protensão e a influência da rigidez dos pilares na retenção da protensão. A investigação foi conduzida por meio de modelagens em elementos finitos, empregando elementos casca para as lajes e elementos viga para os pilares. No caso das lajes nervuradas, suas mesas foram representadas por elementos casca e as nervuras por elementos viga levando em conta a excentricidade entre seus centros geométricos.
\end{abstract}

Palavras-Chave: Lajes Lisas; Análise Paramétrica; Concreto Protendido; Elementos Finitos.

\begin{abstract}
Concrete flat slabs prestressed with unbounded greased strands have been used in building floors over the last years. This structural solution is ideal when the columns are regularly distributed. In addition, it is known that prestressed floors have some technical advantages when compared to the traditional solution in reinforced concrete, mainly in cases of large spans and when lighter elements are required. The objective of the present work is to propose practical criteria for the design of flat slab prestressed concrete floors, for the cases of uniform thickness slabs and waffle slabs, aiming at complying with serviceability limit state. A parametric study was carried out to analyze the stress distribution in prestressing load introduction zones and the influence of the columns in retaining prestressing loads. The investigation was conducted using finite element models in which shell and frame elements were used to represent the slabs and the columns. For the case of waffle slabs, flanges and webs were modeled with shell and frame elements, respectively, taking into consideration the eccentricity between these two elements.
\end{abstract}

Keywords: Flat Slab; Parametric Analysis; Prestressed Concrete, Finite Elements. 


\section{Introdução}

Projetar corretamente lajes protendidas pode contribuir significativamente para a economia e a estética das edificações. Como resultado, esse tipo de laje tem sido empregado com frequência nos pavimentos de edificações nos últimos anos, graças a um número considerável de experiências feitas pelo mundo e a novas concepções arquitetônicas.

Com o intuito de fornecer critérios práticos para o projeto desses pavimentos, foi realizada uma análise numérica do comportamento de pavimentos constituídos de lajes lisas de concreto protendido e os resultados são apresentados neste trabalho. Foram analisadas as tensões, geradas pela componente longitudinal da força de protensão (interpretada como um carregamento externo equivalente), em determinados pontos de controle.

Numa primeira etapa do estudo foi realizada uma análise do comportamento geral das tensões em lajes maciças e nervuradas sem considerar a influência dos pilares. A otimização das tensões provenientes da parcela P/A será a través de um coeficiente que represente melhor estas tensões. O coeficiente $\square$ relaciona as tensões máximas e mínimas nas zonas de regularização das tensões no interior das lajes com a tensão de protensão aplicada na ancoragem. Na segunda etapa foi incluída a contribuição da rigidez dos pilares, para o mesmo estudo, avaliando a força retida nos pilares na sua contribuição na distribuição das tensões.

Foi utilizado o programa computacional SAP 2000 para desenvolver modelos de elementos finitos, utilizando elementos tipo casca para modelar a laje, o qual considera forças no plano nos locais onde a protensão é aplicada, e também considera a contribuição das colunas que são modeladas usando elementos tipo viga. Os resultados do comportamento das tensões atuantes, geradas pelo efeito da protensão como carregamento externo equivalente, são mostrados proporcionando uma comparação entre a solução de programa de computador, muitas vezes utilizado na prática, e a solução encontrada nos cálculos simplificados.

\section{Metodologia da análise numérica}

A primeira análise realizada avalia a relação entre tensão máxima (omax) e tensão de protensão (бp), que será chamado de omax e a relação da tensão mínima (omin) respeito à tensão de protensão (op) que será chamado de Фmin. A tensão de protensão empregada no presente estudo, tanto para lajes maciças como nervuradas, é $1 \mathrm{kN} / \mathrm{cm}^{2}$.

$$
\begin{gathered}
\phi_{\max }=\frac{\sigma_{\max }}{\sigma_{\mathrm{P}}} \\
\phi_{\min }=\frac{\sigma_{\min }}{\sigma_{\mathrm{P}}}
\end{gathered}
$$

Foram criados modelos de lajes lisas e nervuradas com determinados números de painéis e diferentes larguras da faixa de concreto, onde a força de protensão é aplicada, sendo possível encontrar os valores da relação de tensões $(\Phi)$ nos diferentes pontos de análise na laje.

As propriedades mecânicas são a resistência característica à compressão fck $=35 \mathrm{MPa}$, o módulo de deformação longitudinal do concreto $\mathrm{E}=28160 \mathrm{MPa}$ e o coeficiente de Poisson $\mathrm{u}=0,2$.

A análise é feita considerando a protensão aplicada como carga equivalente nas faixas de concreto, com a largura destas faixas relacionada com o comprimento do vão $(\mathrm{L}=10 \mathrm{~m})$. A largura das faixas estudadas são L/4, L/8, L/20 e L/40. Torna-se importante salientar que, em todos os modelos, o carregamento a ser aplicado na estrutura é o carregamento equivalente da força de protensão sem considerar seu peso próprio. Consideraram-se as lajes com concreto não fissurado e comportamento elástico linear.

\subsection{Estudo Paramétrico de Lajes Maciças}

A laje em estudo é uma laje maciça de $20 \mathrm{~cm}$ de espessura com vão típico de $10 \mathrm{~m}$ apoiada diretamente sobre pilares e submetida à tensão de protensão de $1 \mathrm{kN} / \mathrm{cm}^{2}$. Os estudos preliminares concentraram-se na análise da distribuição das tensões causadas pela componente longitudinal da força de protensão que atua no plano da laje. Inicialmente, a análise é feita sem considerar a rigidez dos pilares. Em seguida, foram analisados vários casos com pilares de diferente rigidez a flexão.

\subsubsection{Distribuição das Tensões sem Influência dos Pilares:}

O objetivo desta etapa do estudo é obter o coeficiente $\square$ que relaciona as tensões máximas e mínimas nas zonas de regularização das tensões das lajes com a tensão de protensão aplicada na ancoragem para diferentes larguras de faixa de concreto. As tensões foram avaliadas nas seções $A, B$ e C. 
a) Modelo 1 - Laje com painel isolado

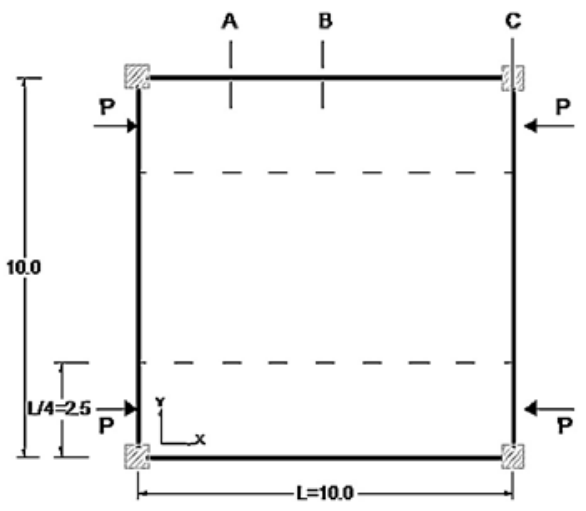

Figura 1 - Laje de referência para o modelo 1 (a) e distribuição de tensões Sx para carregamento de protensão aplicado em uma faixa de 2,5m (b).

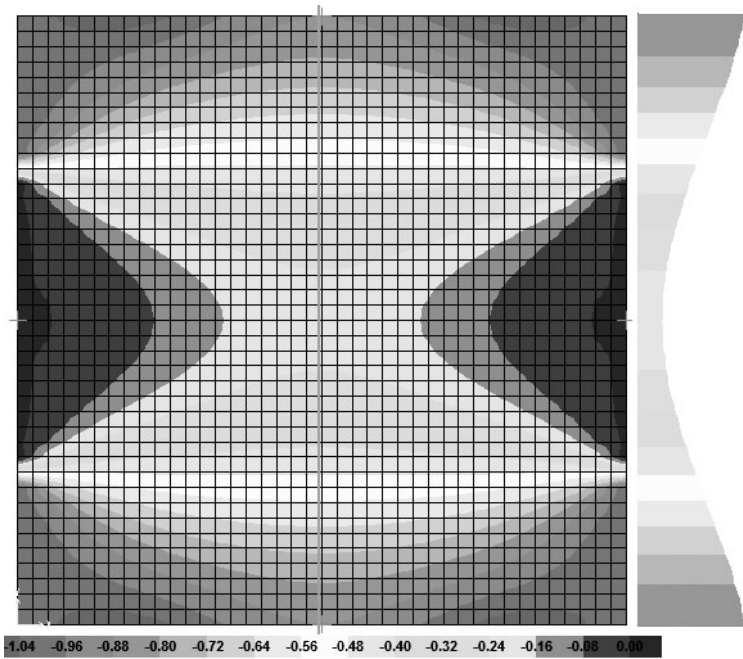

Na Figura 2 são plotados os valores das tensões mínimas e máximas e sua relação com a tensão da protensão $(\Phi)$ para as diferentes larguras de faixas nas seções $A$ e $B$ e mostram-se as equações para a obtenção do coeficiente $\Phi$ calculado em função da largura da faixa de protensão.

Figura 2 - Relação entre a tensão máxima, mínima e a tensão gerada pela força de protensão para as seções A e B.

\section{Seção A}

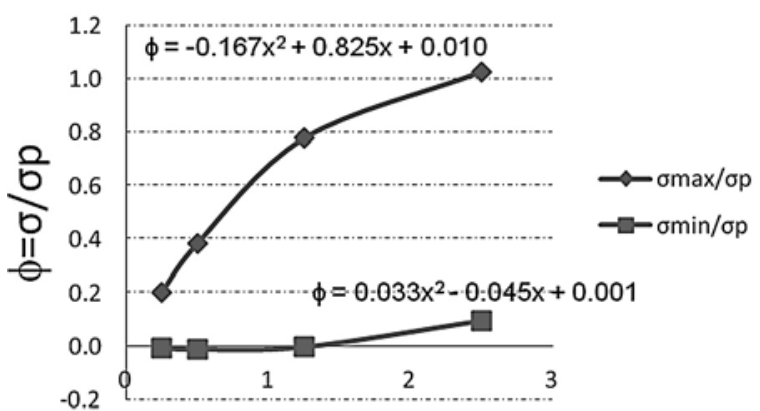

Largura faixa de protensão

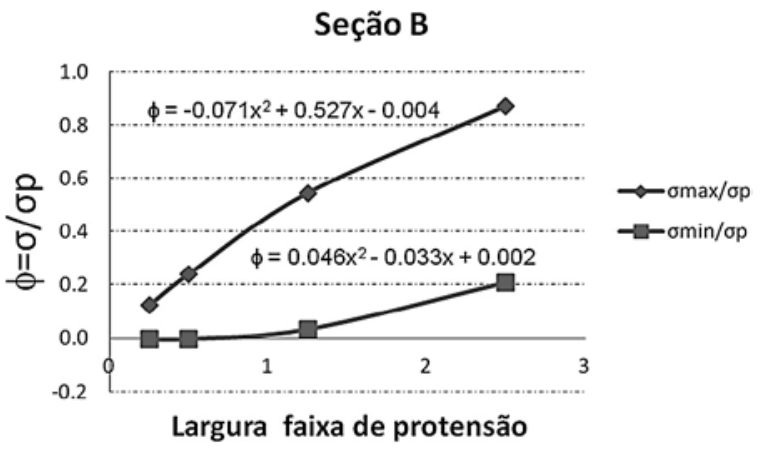

b) Modelo 2 - Dois painéis de laje

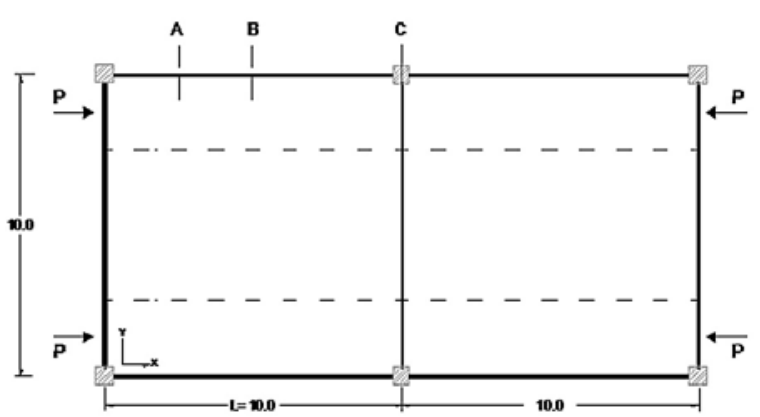

Figura 3 - Laje de referência para o modelo 2 (a) e distribuição de tensões Sx para carregamento de protensão aplicado em uma faixa de 2,5m (b).

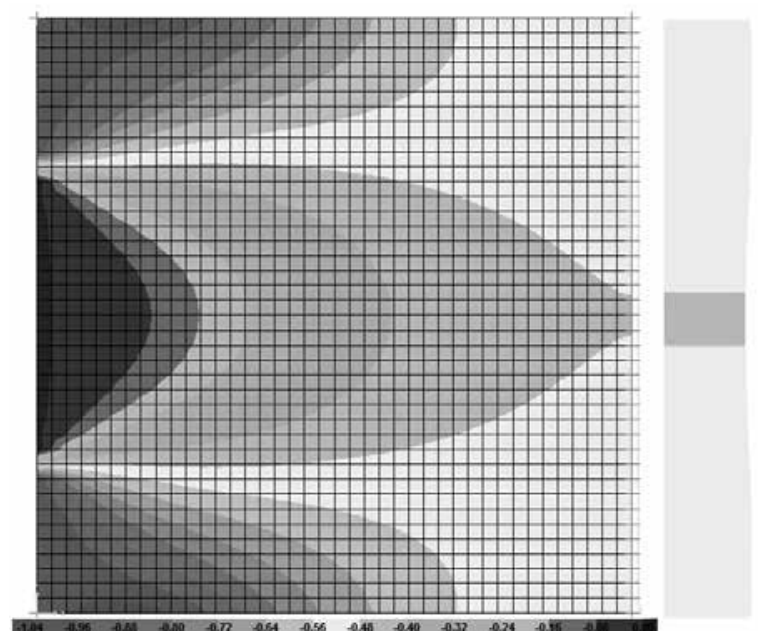

Os resultados do coeficiente $\Phi$ estão apresentados na Figura 4, o qual apresenta os valores das tensões máximas e mínimas com relação à tensão de protensão para as seções $A$, B e $C$. 
Figura 4 - Relação entre a tensão máxima, mínima e a tensão gerada pela força de protensão para as seções A e B.

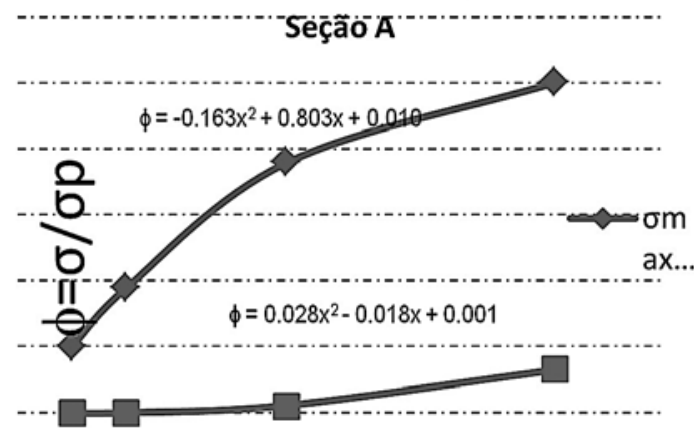

\section{Largura faixa de protensão}

\section{Seção B}

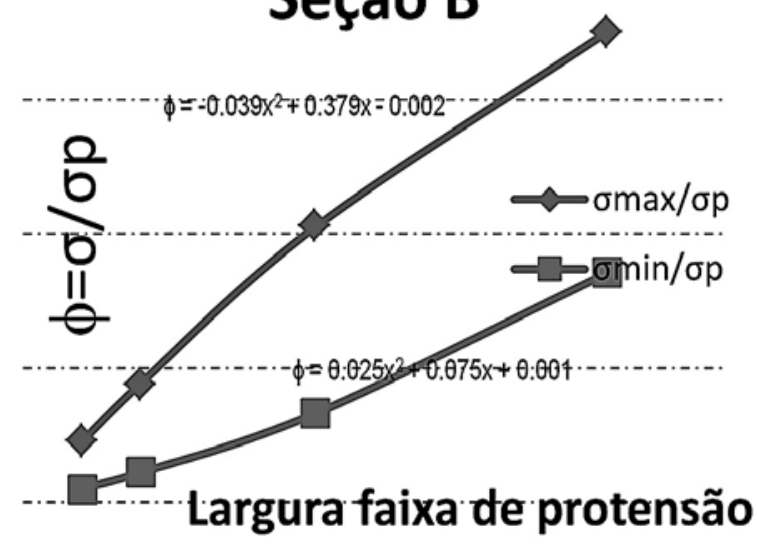

Seção C

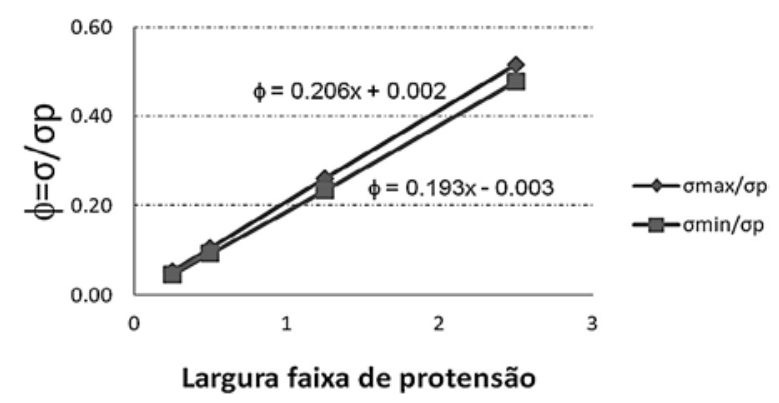

c) Modelo 3 - Quatro painéis de laje adjacentes

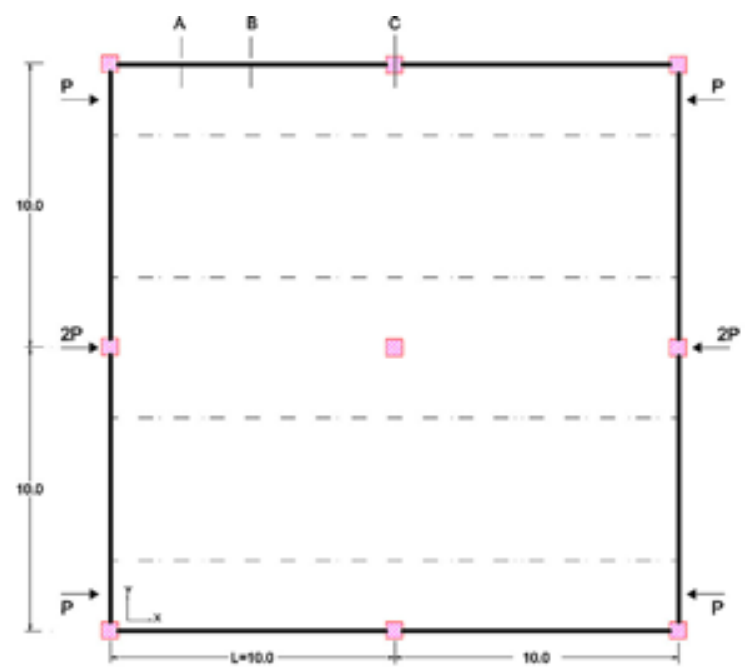

Figura 5 - Laje de referência para o modelo 3 (a) e distribuição de tensões Sx para carregamento de protensão aplicado em uma faixa de $2,5 \mathrm{~m}$ (b).

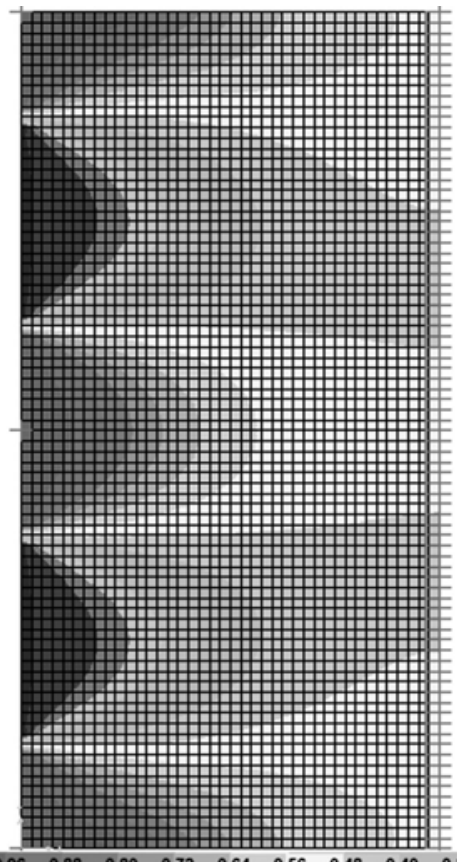

Os valores calculados do coeficiente $\Phi$ para as seções A e B são apresentados na Figura 6.

Figura 6 - Relação entre a tensão máxima, mínima e a tensão gerada pela força de protensão para as seções A e B.
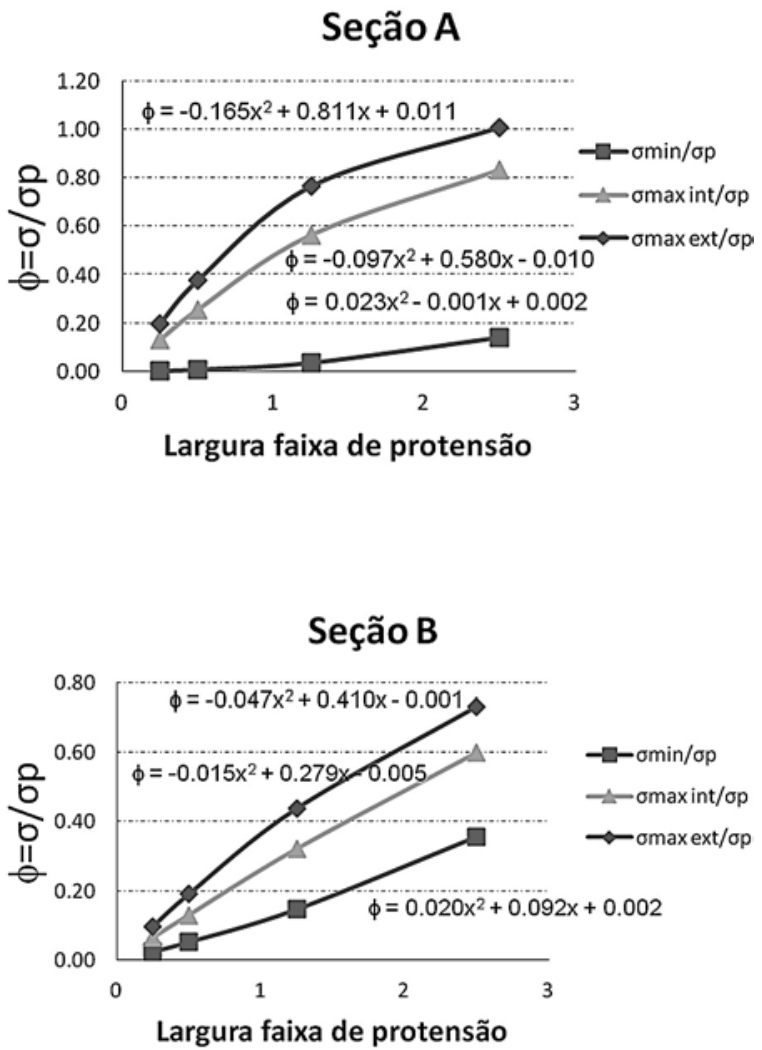
Deste primeiro estudo para lajes maciças pode-se conferir o princípio de Saint Venant, o qual afirma que, em uma seção, a uma distância determinada do comprimento da laje a distribuição das tensões é praticamente uniforme. Isso pode ser notado nos gráficos da distribuição das tensões nos modelos (Figuras 1b, 3b e 5b), onde se observa que a tensão a partir da seção $C$ já é uniforme em todos seus pontos independentemente da largura de aplicação da protensão. Na seção C, no caso das lajes contínuas, a tensão máxima exterior (tensão máxima que surge nos extremos das lajes) difere da tensão máxima interior (tensão máxima que surge na continuidade das lajes) em aproximadamente $14 \%$, independentemente do número de painéis. A diferença entre tensões máximas e mínimas na seção C é de aproximadamente $20 \%$. Desta parte do estudo obtiveram-se equações para calcular o coeficiente $\Phi$ para lajes maciças protendidas. O gráfico ilustrado na Figura 2 fornece valores de $\Phi$ para modelos de laje isolada. $O$ gráfico da Figura 4 fornece valores de $\Phi$ para laje de dois painéis, a qual tem uma diferença mínima ( 6\%) com relação aos gráficos da Figura 6 que fornecem valores de $\Phi$ para lajes de painéis contínuos. Segundo o estudado, o coeficiente $\Phi$ tem aplicação nas seções próximas à aplicação da carga de protensão, já para seções mais afastadas, como a seção $C$, as tensões são uniformes e não ficam afetadas por este coeficiente.

\subsubsection{Distribuição das Tensões com Influência dos Pilares}

a) Coeficiente Aproximado de Rigidez (K):

Nesta segunda etapa estuda-se a influência da rigidez dos pilares sobre as tensões nas lajes. Para tanto, é feita uma análise da relação da rigidez entre lajes e pilares, com a finalidade de obter a força de protensão $(F)$ retida em cada pilar e a partir desta relação fazer uma nova avaliação das tensões na laje, descontando a forca $F$ da força total $(P)$ aplicada na laje. A força $F$ retida nos pilares esta regida pela equação 3 a seguir.

$$
\mathbf{F}=\mathbf{K}\left(\frac{\mathrm{EI}_{\mathrm{p}}}{\mathbf{L}_{\mathrm{p}}^{3}}\right) \cdot \boldsymbol{\delta}
$$

onde:

K é o coeficiente de rigidez

$\delta$ é o deslocamento do topo do pilar

E é o módulo de elasticidade.

IP é o momento de inércia do pilar.

LP é o comprimento do pilar.

As condições de apoio podem simular o caso de uma laje com extremidades engastadas, cujo coeficiente $\mathrm{K}$ é 12 , ou uma laje simplesmente apoiada, com $\mathrm{K}$ igual a 3 . O coeficiente de rigidez $\mathrm{K}$ será vinculado, através de uma equação, com um novo parâmetro $(\mathrm{G})$ o qual relaciona a rigidez entre lajes e pilares, como mostrado na eq. 4 a seguir:

$$
\mathbf{G}=\frac{\left(\mathbf{E I}_{\mathbf{L}} / \mathbf{L}_{\mathbf{L}}\right)}{\left(\mathbf{E \mathbf { I } _ { \mathbf { P } } / \mathbf { L } _ { \mathbf { P } } )}\right.}
$$

onde:

LL é a distância entre pilares.

IL é o momento de inércia para lajes.

E é o módulo de elasticidade.

Foram modelados pórticos com diferentes relações de rigidez laje-pilar (G), como mostrado na Figura 7. Um mesmo carregamento equivalente de protensão de 100 kN é aplicado a cada pórtico. Os modelos são feitos com a mesma rigidez do pilar e variando a rigidez de laje.

Figura 7 - Deformação e diagrama de cortante para laje de rigidez à flexão nula (a), laje de rigidez à flexão intermediária (b) e laje infinitamente rígida(c).

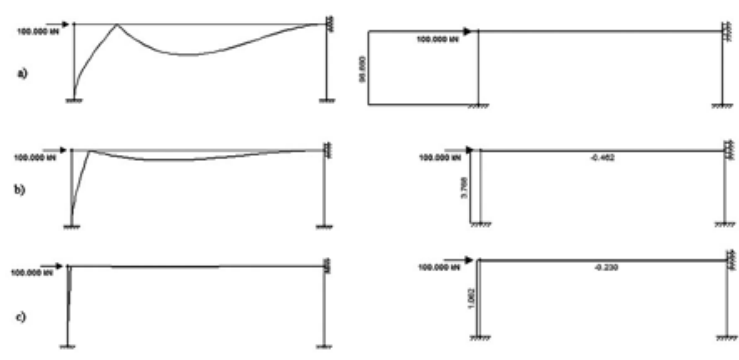

Na Figura 7a têm-se uma laje praticamente sem rigidez à flexão. Para um fator de rigidez entre lajes e pilares $\mathrm{G}=0$ o coeficiente $\mathrm{K}=3$. Caso de uma laje simplesmente apoiada. $O$ inverso ocorre na figura $7 \mathrm{~b}$, para $\mathrm{G}=48$ obteve-se o coeficiente $\mathrm{K}=11$,8. Aproxima do caso de uma laje com extremidades engastadas. Conforme a Figura 8, foram plotados os valores de $\mathrm{G}$ e K para diferentes relações de rigidez, assim é obtida a Equação 5 , a qual reflete estes valores. 
Figura 8 - Curva de tendência que relaciona o fator de rigidez entre lajes e pilares $(G)$ e o coeficiente aproximado de rigidez $(k)$.

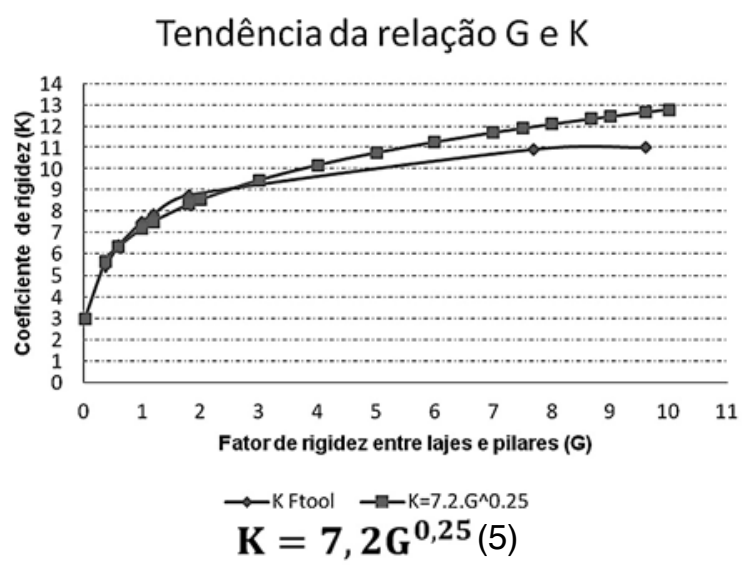

Esta equação é válida para $\mathrm{G} \geq 0,03$, para os quais os valores de $\mathrm{K}$ são maiores a 3 (caso engastadorotulado) e para valores de $G \leq 7,5$, para os quais os valores de K são menores a 12 (caso biengastado).

b) Coeficiente Aproximado de Rigidez (K):

Obtido o valor de K é possível calcular que porcentagem da força de protensão aplicada (P) fica retida nos pilares dependendo da relação de rigidez. Para pilares com rigidez baixa ou media o deslocamento axial $\delta$ é calculado para o pilar analisado com a Equação 6:

$$
\delta=\frac{\mathrm{PL}}{\mathrm{EA}}(6)
$$

Onde:

P é a componente longitudinal da força de protensão.

Lé a distância de influência da força de protensão na laje analisada.

E é o módulo de elasticidade.

A é a área da seção da laje.

Figura 9 - Protótipo dos pórticos laje-pilar

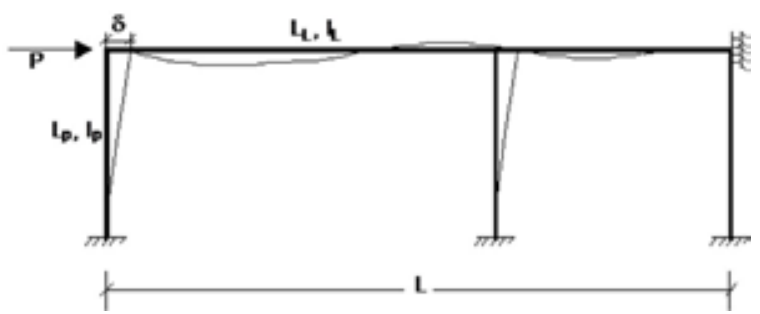

Com os valores do deslocamento $\delta$ e o coeficiente $\mathrm{K}$, é calculado o valor da força $\mathrm{F}$ retida em cada pilar em análise, conforme a equação 3 .

c) Cálculo Simplificado das tensões

Nas seções onde as tensões a serem avaliadas ficam perto do ponto de aplicação de carga o coeficiente $\Phi$ é utilizado. Para calcular as tensões nos diferentes pontos de controle é apenas multiplicar este fator $\Phi$ por a tensão de protensão $\sigma p$ (componente da força longitudinal de protensão $P$ entre a seção transversal da laje). A força $P$, é a forca que passa na laje em cada ponto de análise descontando a força retida nos pilares.

$$
\sigma_{i}=\phi \frac{P}{A_{P}}
$$

Nas outras seções o coeficiente $\Phi$ não tem efeito, devido ao fato de que as tensões já são uniformes. Aqui é empregada a tensão media ( $\sigma \mathrm{m})$ que relaciona a força total $(\mathrm{Pt})$ aplicada na estrutura entre a área transversal total da laje (At).

$$
\sigma_{\mathrm{i}}=\mathrm{P}_{\mathrm{t}} / \mathrm{A}_{\mathrm{t}(8)}
$$

\subsection{Estudo Paramétrico de Lajes Nervuradas}

Da mesma maneira como foi feito o cálculo do coeficiente $\Phi$ para lajes maciças é realizado o cálculo do coeficiente para lajes nervuradas nas mesmas seções. Constata-se novamente o principio de Saint Venant no caso das lajes nervuradas. Obtêm-se também gráficos que ajudaram na escolha do coeficiente $\Phi$. Constata-se que os resultados do coeficiente $\Phi$ utilizando lajes nervuradas forneceram resultados com uma percentagem mínima de diferença (4\%) em relação aos obtidos nos modelos de lajes maciças. O coeficiente $\Phi$ tem aplicação nas seções próximas à aplicação da carga de protensão.

\subsection{Exemplo De Aplicação e Análise dos Resultados}

Neste exemplo as lajes foram analisadas através de cálculos simplificados, com ajuda de planilhas Excel, com procedimentos já descritos. Depois, as lajes foram analisadas usando o programa de análise de estruturas SAP2000, para criar modelos estruturais de forma rápida. A comparação entre estas duas análises irá fornecer um meio para verificar o projeto. 
Figura 10 - Planta de forma destacando as faixas onde a força de protensão é aplicada.

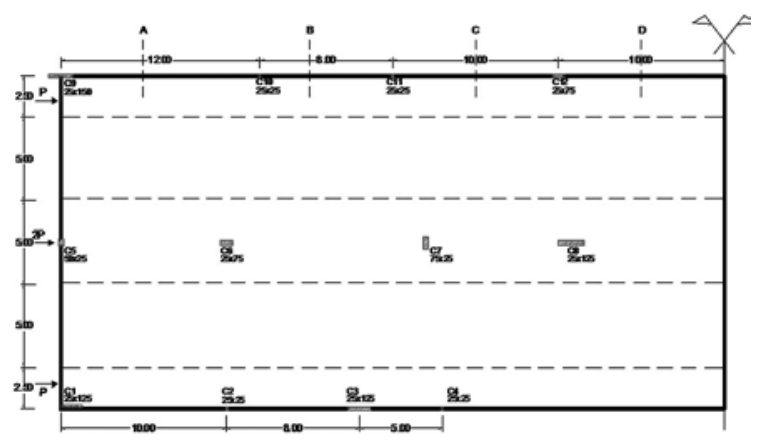

\subsubsection{Cálculos através de Processos Simplificados:}

a) Cálculo da Força de Protensão Retida em Cada Pilar (F):

Para fins do exemplo será obtida a força $F$ no pilar C9 $(0.25 \mathrm{~m} \times 1.50 \mathrm{~m})$. O primeiro coeficiente calculado é o coeficiente $G$ seaundo a equação 4 transcrita $\in \mathrm{G}=\frac{\left(\mathrm{I}_{\mathrm{L}} / \mathrm{L}_{\mathrm{L}}\right)}{\left(\mathrm{I}_{\mathrm{P}} / \mathrm{L}_{\mathrm{P}}\right)}=0,023$

Como o coeficiente $\mathrm{G}$ está fora do intervalo de $0,03 \leq \mathrm{G} \leq 7,5, \mathrm{~K}$ adota o valor de 3 . O deslocamento axial $\delta$ é calculado multiplicando a componente longitudinal da força de protensão com a rigidez axial da laje usando a equação 6 transcrita em baixo.

$$
\delta=\frac{P * L}{E * A}=0,43 \mathrm{~cm}
$$

Para pilares com rigidez media ou alta é necessário um processo iterativo para o cálculo do valor de F. Com o deslocamento $0,43 \mathrm{~cm}$, para uma força $P$ de $3000 \mathrm{kN}$, é obtido um valor de força que passa na laje $\mathrm{Pi}$. Com este valor é obtido um novo valor

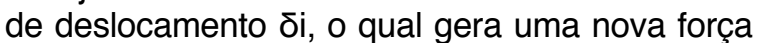
de retenção da protensão no pilar Fi. Desta forma, inicia-se um processo iterativo com a finalidade de obter a força mais aproximada retida no pilar $F$.

Tabela 1 - Força na laje e no pilar $(\mathrm{kN})$ para análise dos pilares $\mathrm{C} 1$ e C9.

\begin{tabular}{|c|c|c|c|c|c|}
\hline \multicolumn{3}{|c|}{ Pilar C1 } & \multicolumn{3}{c|}{ Pilar C9 } \\
\hline $\begin{array}{c}\text { Força } \\
\text { Laje } \\
(\mathbf{k N})\end{array}$ & $\begin{array}{c}\text { Força } \\
\text { Pilar (kN) }\end{array}$ & $\delta$ & $\begin{array}{c}\text { Força Laje } \\
(\mathbf{k N})\end{array}$ & $\begin{array}{c}\text { Força Pilar } \\
(\mathrm{kN})\end{array}$ & $\delta$ \\
\hline 3000 & 533 & 0.43 & 3000 & 1217 & 0.43 \\
\hline 2296 & 539 & 0.33 & 1783 & 723 & 0.25 \\
\hline 2461 & 578 & 0.35 & 2277 & 924 & 0.32 \\
\hline 2422 & 569 & 0.34 & 2076 & 842 & 0.29 \\
\hline 2431 & 533 & 0.35 & 2158 & 875 & 0.31 \\
\hline 2429 & 570 & 0.35 & 2125 & 862 & 0.3 \\
\hline 2430 & 570 & 0.35 & 2138 & 867 & 0.3 \\
\hline- & - & - & 2133 & 865 & 0.3 \\
\hline- & - & - & 2135 & 866 & 0.3 \\
\hline- & - & - & 2134 & 866 & 0.3 \\
\hline
\end{tabular}

Com os valores do deslocamento $\delta$ e o coeficiente $\mathrm{K}$, é calculado o valor da força $\mathrm{F}$ retida no pilar em análise. Por tanto, pode-se obter a força $\mathrm{P}$ que passa na laje, como mostrado na Tabela 2.

Tabela 2 - Quadro arranjado para a obtenção das forças retidas nos pilares

\begin{tabular}{|c|c|c|c|c|c|c|c|c|c|c|}
\hline Pilar & $\mathrm{L}_{1}(\mathrm{~cm})$ & $I_{r}(\mathrm{~cm})$ & $P(k N)$ & L. $(\mathrm{cm})$ & $\mathrm{L}_{\mathrm{L}}(\mathrm{cm})$ & $\mathrm{I}_{\mathrm{p}}(\mathrm{cm})$ & 8 & G & K & \\
\hline $\mathrm{Cl}$ & & & & 4000 & & 4069010 & 0,35 & 0 & 3 & 70 \\
\hline C2 & 800 & 775 & 2430 & 3000 & 333333 & 32552 & 0.26 & 3,5 & 9,9 & 11 \\
\hline C3 & & & 2930 & 2200 & 333333 & 7031250 & 0,19 & & 3 & 542 \\
\hline Ca & 1700 & 275 & 430 & 1700 & 333333 & 32552 & 0,15 & 1,7 & 8,2 & 5 \\
\hline Cs & 1000 & 275 & 6000 & 4000 & 666667 & 65104 & 0,43 & 2,8 & 9,3 & 35 \\
\hline C6 & 1200 & & 600 & 3000 & 666667 & 878906 & 0,32 & 0,2 & & 177 \\
\hline C7 & & 275 & 6000 & 1800 & 666667 & 97656 & 0,19 & 2,3 & 8,9 & 23 \\
\hline $\mathrm{C} 8$ & 1000 & 275 & 6000 & 1000 & 666667 & 7031250 & 0,11 & 0 & 3 & 304 \\
\hline c9 & 1200 & 275 & 2134 & 4000 & 333333 & 7031250 & 0,3 & 0 & 3 & 866 \\
\hline $\mathrm{Cl} 0$ & & & 2134 & 2800 & 333333 & 32552 & 0,21 & 3,5 & 9,9 & 9 \\
\hline $\mathrm{Cl1}$ & 1000 & 275 & 2134 & 2000 & 333333 & 32552 & 0,15 & 2,8 & 9,3 & 6 \\
\hline $\mathrm{Cl} 2$ & 1000 & 275 & 2134 & 1000 & 333333 & 878906 & 0,08 & 0.1 & 4,1 & 37 \\
\hline
\end{tabular}

b) Valor do Coeficiente de Relação de Tensões $(\Phi)$ :

Este coeficiente tem influência somente em determinado comprimento da laje, como é o caso da seção A do presente exemplo. Os valores destes coeficientes foram obtidos com as equações da Figura 6.

$$
\text { Фmax ext }=0,73 \quad \underset{0,36}{\Phi \max } \text { int }=0,60 \quad \text { Фmin }=
$$

c) Cálculo das Tensões na Seção A:

Esta seção por se encontrar próximo da aplicação da protensão, a tensão de análise é a tensão de protensão op a qual é afetada pelo coeficiente $\Phi$, conforme equação 7 . Serão avaliadas as tensões nos pontos de controle T9 ,t5 e T5.

Figura 11 - Seções e pontos de controle das tensões.

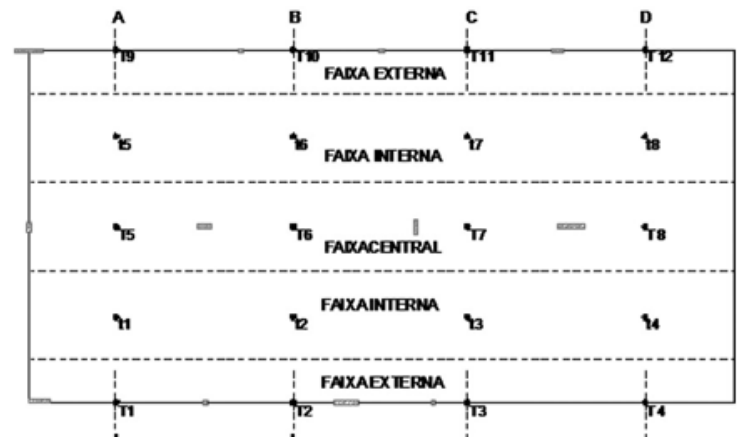

O ponto de controle T9 tem próximo a ele um pilar com elevada rigidez. Foi necessário o processo iterativo descrito acima para obter a força $P=2134 k N$. Com a tensão de protensão $(\sigma P)$ multiplicada pelo coeficiente $\Phi$ max ext é obtida a tensão no ponto T9: 


$$
\begin{gathered}
\sigma_{\mathrm{P}}=\frac{-2134}{(20)(250)}=-0,427 \mathrm{kN} / \mathrm{cm}^{2} \\
\sigma_{\mathrm{T} 9}=0,73 * 0,43=0,312 \mathrm{kN} / \mathrm{cm}^{2}
\end{gathered}
$$

O ponto de controle T5 encontra-se próximo a um pilar com rigidez menor comparada com o pilar $\mathrm{C} 9$

$$
\begin{aligned}
& \sigma_{\mathrm{P}}=\left(\frac{-5965}{20 * 500}\right)=-0,596 \mathrm{kN} / \mathrm{cm}^{2} \\
& \sigma_{\mathrm{T} 5}=0,60 * 0.596=0,358 \mathrm{kN} / \mathrm{cm}^{2}
\end{aligned}
$$

O ponto t5 encontra-se entre o pilar C9 e C5. Este ponto de controle é um ponto médio das tensões de protensão em T9 e T5, afetado pelo coeficiente $\Phi$ min.

$$
\begin{gathered}
\sigma_{\mathrm{P}}=\left(\frac{\sigma_{\mathrm{T} 9}+\sigma_{\mathrm{T} 5}}{2}\right)=-0,512 \frac{\mathrm{kN}}{\mathrm{cm}^{2}} \\
\sigma_{\mathrm{t} 5}=\emptyset_{\min } \cdot \sigma_{\mathrm{P}}=0,183 \mathrm{kN} / \mathrm{cm}^{2}
\end{gathered}
$$

Assim como foram encontradas as tensões na seção $A$, foram calculadas as demais tensões nos outros pontos de controle.

\subsubsection{Cálculo por Meio da Análise de El- ementos Finitos}

Nesta etapa os cálculos foram realizados através do programa de cálculo estrutural SAP2000, que utiliza o método dos elementos finitos. A Figura 12 mostra a laje do exemplo modelada com elementos casca de $25 \mathrm{~cm} \times 25 \mathrm{~cm}$ e com elementos viga para as nervuras.

Figura 12 - Configuração deformada da estrutura, modelada no programa SAP2000.

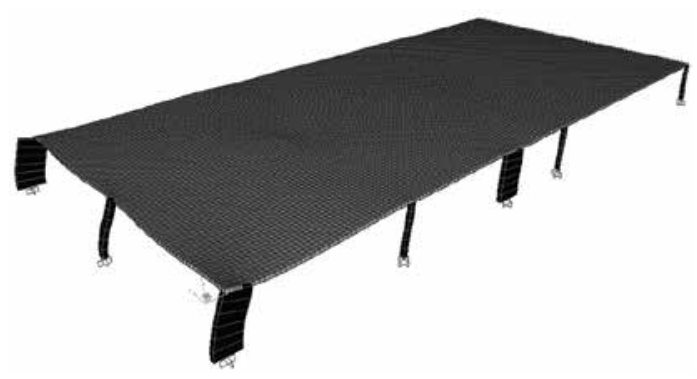

Da modelagem com elementos finitos encontrase a força cortante retida em cada pilar e verificam-se na Tabela 3 que os valores da força na laje são inferiores na análise feita com o método simplificado $(\sim 4 \%)$ aos obtidos com o programa SAP2000.
Tabela 3 - Quadro comparativo das forças obtidas numericamente e do SAP2000 com referência à forca retida em cada pilar.

\begin{tabular}{|c|c|c|c|c|c|c|}
\hline \multirow{2}{*}{ Pilar } & \multirow{2}{*}{$\begin{array}{c}\text { bxh } \\
\text { (cm }\end{array}$} & \multicolumn{2}{|c|}{$\begin{array}{c}\text { \% de P retida no } \\
\text { pilar }\end{array}$} & \multicolumn{2}{|c|}{$\begin{array}{c}\text { Força P que passa à Laje } \\
\text { (kN) }\end{array}$} & $\begin{array}{c}\text { P } \\
\text { cal/ }\end{array}$ \\
\cline { 3 - 6 } & & SAP & Calculada & Calculada & SAP & $\begin{array}{c}\text { P } \\
\text { SAP }\end{array}$ \\
\hline C1 & $25 \times 125$ & $4 \%$ & $5 \%$ & 2430 & 2467 & 0.98 \\
\hline C2 & $25 \times 25$ & $0 \%$ & $0 \%$ & 2418 & 2457 & 0.98 \\
\hline C3 & $25 \times 150$ & $3 \%$ & $5 \%$ & 1876 & 2058 & 0.91 \\
\hline C4 & $25 \times 25$ & $0 \%$ & $0 \%$ & 1871 & 2053 & 0.91 \\
\hline C5 & $50 \times 25$ & $0 \%$ & $0 \%$ & 5965 & 5977 & 1 \\
\hline C6 & $25 \times 75$ & $1 \%$ & $1 \%$ & 5788 & 5860 & 0.99 \\
\hline C7 & $75 \times 25$ & $0 \%$ & $0 \%$ & 5766 & 5844 & 0.99 \\
\hline C8 & $25 \times 150$ & $2 \%$ & $3 \%$ & 5461 & 5657 & 0.97 \\
\hline C9 & $25 \times 150$ & $6 \%$ & $7 \%$ & 2134 & 2266 & 0.94 \\
\hline C10 & $25 \times 25$ & $0 \%$ & $0 \%$ & 2125 & 2257 & 0.94 \\
\hline C11 & $25 \times 25$ & $0 \%$ & $0 \%$ & 2119 & 2251 & 0.94 \\
\hline C12 & $25 \times 75$ & $0 \%$ & $0 \%$ & 2082 & 2216 & 0.94 \\
\hline
\end{tabular}

Quanto às tensões, discrepâncias entre resultados dos cálculos simplificados e análise no SAP são resumidas na Figura 13.

Figura 13 - Tensões obtidas dos cálculos simplificados e do SAP2000 para todos os pontos de control

\section{Grafico Comparativo de Tensões}

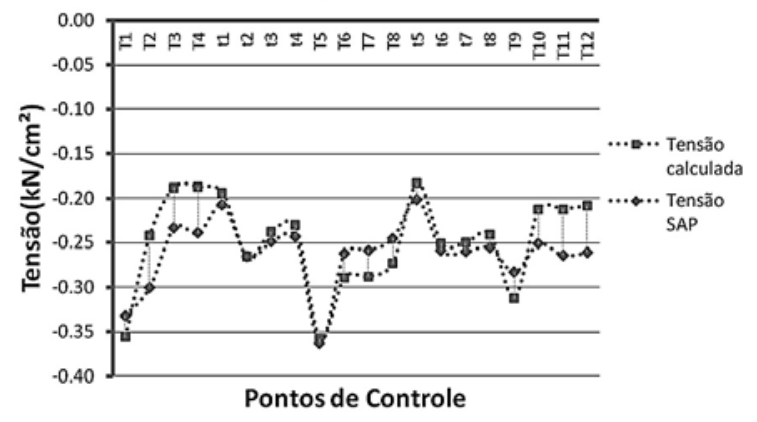

\section{Conclusões}

As tensões obtidas com o procedimento simplificado foram satisfatórias em comparação com os resultados obtidos com a modelagem no programa computacional SAP2000. Através dos estudos realizados e pelos exemplos analisados, pode-se afirmar que com a aplicação dos coeficientes encontrados $(\Phi)$ e considerando a influência dos pilares, de uma maneira muito simples, encontram-se resultados bons para a estimativa dos valores das tensões máximas e mínimas. Com base nos gráficos e tabelas obtidos, foi observado também que o método apresentado é apropriado para lajes maciças. Em geral, o método simplificado fornece valores para tensões superiores às fornecidas pelo programa SAP2000, que é uma solução a favor da segurança.

A partir dos quadros e das figuras apresentadas, consegue-se avaliar a distribuição de tensões das lajes nervuradas, para posteriormente fazer uma comparação com a laje maciça correspondente. Constata-se que os resultados do coeficiente $\Phi$ 
utilizando lajes nervuradas fornecem resultados semelhantes aos obtidos nos modelos de lajes maciças.

Por fim, conseguiu-se estabelecer critérios práticos para o projeto de lajes lisas protendidas, maciças ou nervuradas, através de uma metodologia pratica e simplificada, visando fornecer subsídios para o calculo estrutural de maneira confiável.

\section{Referências}

AALAMI, B.O.; BOMMER, A. Design Fundamentals of Post-Tensioned Concrete Floors. 1. ed. Post-Tensioning Institute, 1999. 178p.

LIN, T. Y. Design on Prestressed Concrete Structures. 2 ed. New York: Wiley, 1963. 614p.

THE CONCRETE CENTRE. Post-tensioned Concrete Floors. 2008. 21p.

ASSOCIAÇÃO BRASILEIRA DE NORMAS TÉCNICAS. NBR 6118/2007. Projeto de estruturas de concreto - Procedimento. Rio de Janeiro, 2007.

BUILDING CODE REQUIREMENTS FOR STRUCTURAL CONCRETE. ACI 318M-05. American Concrete Institute. Michigan: Farmington Hills, 2005.

POST-TENSIONING INSTITUTE. Post-Tensioning Manual. 6. ed. U.S.A., 1995. 345p.

RECOMMENDATIONS FOR CONCRETE MEMBERS PRESTRESSED WITH UNBOUNDED TENDONS. ACl 423.3R-96. Detroit, 1983.

KHAN, S.; WILLIAMS, M. Post-Tensioned Concrete Floors. Great Britain: Butterworth Heinemann. 1995. 312pg.

COMPUTERS AND STRUCTURES, INC., CSI Analysis Reference Manual For SAP2000, ETABS, and SAFE. Berkeley, California, 2008. 
